\title{
O programa saúde na escola no combate de doenças precoces: uma revisão narrativa
}

\author{
The school health program in the fight against early diseases: a narrative review \\ El programa de salud escolar en la lucha contra las enfermedades tempranas: una \\ revisión narrativa
}

Lázaro Saluci Ramos ${ }^{1 *}$, Cristina Pereira Baiense Viana ${ }^{2}$, Jose Rodrigo Brioli Polonini², Lucas da Silva Saúde ${ }^{2}$, Poliana Nicoli Fontana ${ }^{2}$, Maurício Lopes Spínola ${ }^{2}$, Elba Corrêa de Jesus ${ }^{2}$, Elisangela Moraes Ayres², Manoela Paz da Costa², Eliana Bayerl Moreira Bahiense².

\begin{abstract}
RESUMO
Objetivo: Intenta-se nesta revisão, apresentar o Programa Saúde na Escola (PSE) como alternativa às doenças precoces em crianças e adolescentes, e como o mesmo atua no campo da prevenção. Revisão Bibliográfica: $O$ PSE atua em vários campos da saúde do aluno. Desde a saúde mental, passando pela saúde alimentar até os campos da saúde física. É imprescindível apresentar este programa como a melhor maneira de garantir a saúde das futuras gerações e como o mesmo pode ser fortalecido através de novas propostas de políticas públicas de saúde. O PSE atua em conjunto com a escola e une dois dos principais campos de responsabilidade do Estado, fazendo com que ambos caminhem em conjunto na busca por melhores condições para a sociedade. As atuações de profissionais da saúde na escola se dá através deste programa e inspira novos cuidados e novas práticas. Considerações finais: A literatura existente colabora para apresentar como o PSE atua nas escolas brasileiras e aponta a parte deficitária, onde novas propostas podem ser trabalhadas para melhorar a atuação do programa.
\end{abstract}

Palavras-chave: Saúde, PSE, Saúde do aluno.

\begin{abstract}
Objective: The aim of this review is to present the Health at School Program (PSE) as an alternative to early diseases in children and adolescents, and how it works in the field of prevention. Bibliographic Review: The PSE works in several fields of student health. From mental health, through food health to the fields of physical health. It is essential to present this program as the best way to guarantee the health of future generations and how it can be strengthened through new proposals for public health policies. The PSE works together with the school and unites two of the main fields of responsibility of the State, making them both go together in the search for better conditions for society. The performance of health professionals at the sedá school through this program and inspires new care and new practices. Final considerations: The existing literature collaborates to present how the PSE works in Brazilian schools and points out the deficit, where new proposals can be worked on to improve the performance of the program.
\end{abstract}

Keywords: Health, PSE, Student health.

\section{RESUMEN}

Objetivo: El objetivo de esta revisión es presentar el Programa Salud en la Escuela (PSE) como alternativa a las enfermedades tempranas en niños y adolescentes, y cómo funciona en el campo de la prevención. Revisión bibliográfica: EI PSE trabaja en varios campos de la salud de los estudiantes. Desde la salud mental, pasando por la salud alimentaria hasta los campos de la salud física. Es fundamental presentar este programa como la mejor forma de garantizar la salud de las generaciones futuras y cómo se puede fortalecer a través de nuevas propuestas de políticas públicas de salud. EI PSE trabaja en conjunto con la escuela y une dos de los principales campos de responsabilidad del Estado, haciendo que ambos vayan juntos en la búsqueda de mejores condiciones para la sociedad. El desempeño de los profesionales de la salud en la escuela sedá a través de este programa inspira nuevos cuidados y nuevas prácticas. Consideraciones finales: La literatura existente colabora para presentar cómo funciona el PSE en las escuelas brasileñas y señala el déficit, donde se pueden trabajar nuevas propuestas para mejorar el desempeño del programa.

Palabras clave: Salud, PSE, Salud del estudiante.

${ }^{1}$ Faculdade Multivix (MULTIVIX), Cachoeiro de Itapemirim - ES. *E-mail: lazaro321123@gmail.com

${ }^{2}$ Faculdade Vale do Cricaré (FVC), São Mateus - ES. 


\section{INTRODUÇÃO}

Políticas e programas de saúde pública e educação são essenciais para formar cidadãos e melhorar a qualidade de vida e saúde da população. Um melhor nível de educação está associado a uma população mais saudável, da mesma forma que uma população saudável tem mais probabilidade de aprender com a educação formal e informal. A escola, além de proporcionar conhecimentos sobre saúde, organizados em disciplinas, deve também educar e desenvolver valores e atitudes críticas relacionadas à realidade social e aos estilos de vida, nos processos de aquisição de competências que apoiem a aprendizagem ao longo da vida, e isso fomenta a autonomia e empoderamento na promoção da saúde (LOPES IE, et al., 2018).

Historicamente, a escola tem sido considerada um ambiente onde se inserem os problemas de saúde e existem problemas no cotidiano. No Brasil, diversos modelos têm sido utilizados, desde modelos orientados para a domesticação até modelos orientados para a assistência clínica, bem como propostas recentes para estimular a competência chave e a autonomia de sujeitos condizentes com a promoção da saúde. As campanhas de promoção da saúde tornaram-se uma política de saúde no Brasil, e as escolas são um ambiente propício para a utilização de recursos educacionais para esse fim. Apesar de vigorosos exercícios e ações voltadas à promoção da saúde, os serviços de saúde e as escolas não atendiam o jovem por ser uma pessoa com riscos, vulnerabilidades e necessidades específicas de cuidados (BRASIL EGM, et al., 2017).

É necessário discutir os conceitos que sustentam a ação saudável como prática docente e deixar esses conceitos claros para todos os participantes. O conceito de pedagogia são as diferentes formas de compreender, teorizar e praticar a educação, e expressar a forma de operar e implementar o comportamento educacional. Do ponto de vista da pedagogia, os diferentes conceitos podem ser divididos em duas tendências principais: a pedagogia tradicional e os conceitos anti-hegemonia. É importante notar que várias tendências no pensamento e na prática docente estão próximas de uma tendência ou de outra. Como o primeiro exemplo, temos uma tendência tecnológica baseada na produtividade, e o segundo é um método de ensino histórico crítico, em que a prática social é o ponto de partida e o fim da prática educativa. De uma forma geral, a tendência tradicional pode ser expressa pela passividade do aprendiz, e a passividade do aprendiz não é considerada como sujeito do processo de aprendizagem, ele só recebe informações de uma forma que não condiz com a realidade (CARVALHO FFB, 2015).

As escolas são locais importantes onde a saúde e a educação se encontram, proporcionando amplas oportunidades para serviços de saúde especializados ou cuidados básicos, como diagnóstico clínico e/ou rastreio social e/ou estratégias de recomendação; atividades de educação em saúde e promoção da saúde. No Brasil, o Programa Saúde na Escola (PSE) desenvolvido de acordo com o Decreto Presidencial no 6.286/2007 representa a atual estratégia de saúde na escola. Em relação à alimentação escolar, o país estabelece políticas públicas com essa finalidade desde a década de 1950, mas aprovou a Lei no 11.947/2009 (que define o Programa Nacional de Alimentação Escolar (PNAE), confirmando o direito à alimentação escolar como universal E relacionado à estratégia de promoção da saúde da escola (CASEMIROJP, et al., 2014).

O "Manual Passo a Passo do PSE" desenvolvido em conjunto pelo Ministério da Educação e Saúde descreve oito diretrizes implementadas nas escolas, tais como: tratar a saúde e a educação integral como parte da formação integral e pleno gozo dos direitos humanos dos cidadãos; e fortalecer o Confronto frágil, que pode comprometer o desenvolvimento de toda a escola. Como parte das atividades do programa, a equipe de saúde da família visitará as escolas participantes de forma regular e permanente para avaliar a saúde dos alunos e prestar atendimento ao longo do ano letivo de acordo com as necessidades locais. Por fim, o acompanhamento e avaliação do PSE é da competência da comissão interministerial, mas não existe relatório público sobre essa avaliação. Esta lacuna reforça a importância de iniciativas que apontem para a contribuição do programa para os jovens das escolas que aderem ao PSE (ATALIBA P e MOURÃO L, 2018).

Assim, o presente artigo busca avaliar e revisar através de uma narrativa do PSE de maneira mais aberta, evidenciar as políticas públicas executadas pelo mesmo e suas importâncias na prevenção de doenças precoces nos alunos. É também um objetivo caracterizar pontos necessários para novas implementações ou 
que mereçam uma atenção maior do Estado para reforçar essas políticas. O PSE promove a atuação em conjunto de escola e saúde, o que amplia a margem de atuação do Estado na sociedade. Esta atuação não só produz efeitos imediatos na sociedade, mas também garante uma saúde mais plena e eficaz nas futuras gerações.

\section{REVISÃO BIBLIOGRÁFICA}

A escola é um espaço de formação socioeducativa que pode dar uma contribuição significativa para o ensino das disciplinas de forma plena, integral e saudável. Nesta perspectiva, o contexto escolar é essencial para o desenvolvimento de conhecimentos compartilhados e integração na comunidade. Onde há uma grande proporção da população demonstrando interesse pela ciência com potencial para divulgar informações. A educação em saúde é um importante meio de ampliar o conhecimento sobre as práticas relacionadas ao comportamento saudável dos indivíduos, nesse contexto, os esforços de educação em saúde são persuasivos, pois visam prescrever determinados comportamentos considerados essenciais para prevenir ou minimizar problemas de saúde (GUETERRES ECR, et al., 2017).

As relações entre os setores da educação e da saúde apresentam muitas semelhanças no domínio das políticas públicas, uma vez que se baseiam na universalização dos direitos fundamentais e, por conseguinte, favorecem uma maior proximidade com os cidadãos das diferentes zonas do país. Uma semelhança que historicamente tem sido uma unidade, pelo menos no caso do Brasil, quando na década de 1950 o então Ministério da Educação e Saúde (MES) se dividiu em duas partes: o Ministério da Saúde e o Ministério da Educação e Cultura, possuindo autonomia institucional para se desenvolver e implementação de políticas em suas áreas. Na ocasião, as atividades desenvolvidas pela Secretaria Nacional de Saúde do antigo MES passaram para o Ministério da Saúde (BRASIL, 2009).

Geralmente, os técnicos de saúde têm entendido a educação em saúde escolar como intervenções específicas voltadas para o conteúdo atual relacionado à doença. Por exemplo, técnicos de saúde falam sobre dengue entre crianças e adolescentes na escola porque a epidemia de dengue está devastando a sociedade. Acreditamos que esta seja no mínimo uma intervenção equivocada, pois quando defendemos os Parâmetros Curriculares Nacionais (PCN), conteúdos saudáveis devem constar no currículo para a formação de crianças e adolescentes numa abordagem interdisciplinar e multidisciplinar. Este conteúdo das aulas é o foco de todas as escolas em todos os níveis e séries e está integrado a todas as disciplinas em uma discussão diária do processo de ensino/aprendizagem (FIGUEIREDO TAM, et al., 2010).

O PSE visa integrar e esclarecer permanentemente a educação e a saúde para a melhoria da qualidade de vida da população brasileira. Como consolidar essa postura dentro da escola? É esta a indagação que nos leva a elaborar a metodologia "Agenda de Educação e Saúde", que será implementada como projeto de ensino da escola. O PSE visa contribuir para a formação integral dos alunos por meio de ações de promoção, prevenção e saúde para dar resposta às vulnerabilidades que colocam crianças e jovens da rede pública de ensino em risco para o seu desenvolvimento global. Os beneficiários do PSE são alunos da educação básica, educação e saúde, gestores e profissionais da comunidade escolar e, de forma mais ampla, alunos da Rede Federal de Educação Profissional Técnica e Educação de Jovens e Adultos. (BRASIL, 2018).

As doenças mentais são entendidas como distúrbios na trajetória de vida decorrentes de alterações no desenvolvimento do sistema nervoso e que se revelam na infância. Essa perspectiva enfatiza o papel da escola, pois os professores e a família não se preocupam apenas com a questão da aprendizagem, mas possuem uma condição privilegiada, pois desde que passou a ser obrigatória para todas as crianças e adolescentes brasileiros, esse ambiente tornou-se um local privilegiado de alta concentração de estimulação longitudinal e de grande impacto em todos os aspectos da vida (GARCIA JM, 2016).

As diretrizes para a promoção da alimentação saudável nas escolas foram recentemente introduzidas como um elemento a ser incluído no desenho político pedagógico das unidades escolares. A construção deste projeto envolve a participação dos trabalhadores da educação, bem como da escola e da comunidade local, a fim de conectar a escola com a comunidade para fins de gestão democrática. Nesse sentido, o Ministério da Saúde e o Ministério da Educação são solidariamente responsáveis por garantir a implementação da 
Política Nacional de Alimentação e Nutrição e, portanto, parte da Política Nacional de Promoção da Saúde nas escolas, de acordo com os critérios de implementação do Programa Nacional de Alimentação Escolar (PNAE). Este, por sua vez, visa atender às necessidades nutricionais do aluno durante o período escolar e, neste contexto, promover hábitos alimentares saudáveis (CERVATO-MANCUSO AM, et al., 2013).

\section{Ações do PSE no combate de doenças precoces}

O PSE pode ser um mecanismo importante para avançar por meio de ações de promoção da saúde, e esses avanços só resistem a comportamentos radicais. Destaca-se que essa ação não apenas expressa uma compreensão do que é saúde, mas também a expressa na forma de uma organização do serviço. No entanto, deve-se considerar que as recomendações propostas nem sempre são seguidas ou implementadas. No que se refere à promoção da saúde, observa-se que a compreensão de sua conceituação se reflete em alguns entraves. É importante destacar que, ao longo dos anos, seu conceito foi mudando, com o enquadramento social, político e econômico de cada período (CAVALCANTI PB, et al., 2015).

Dentre os programas institucionais que atuam no âmbito da promoção da saúde, destaca-se o PSE, que, em articulação com a Estratégia Saúde da Família desde 2007, se propõe a integrar e articular setores e atividades voltadas à melhoria da qualidade de vida dos alunos do ensino fundamental. O programa é executado com base em cinco componentes: avaliar o estado de saúde de crianças, adolescentes e jovens no sistema escolar público; medidas de promoção da saúde e prevenção de doenças e questões de saúde; educação de jovens e educação e treinamento vitalício de trabalhadores de saúde; monitorar e avaliar o estado de saúde dos alunos; monitorar e avaliar o plano e suas mudanças sociais (CAVALCANTI, et al., 2015).

O aumento da incidência de sobrepeso (sobrepeso e obesidade) foi reconhecido como uma pandemia. Em pouco mais de 30 anos (1975-2009), essa incidência aumentou entre a população adulta e mais jovem de vários países, principalmente nos países em desenvolvimento, onde aumentou cerca de $50 \%$ em crianças e adolescentes (2-19 anos). No Brasil, de meados da década de 1970 ao final do século 21, o aumento da prevalência de excesso de peso na população infanto-juvenil foi ainda mais acentuado, com meninas e meninos de 5 a 9 anos passando de $8,6 \%$ para $32 \%$ e 10,9\% para $34 \%$. No caso dos adolescentes, esse indicador passou de $7,6 \%$ para $19,4 \%$ nas mulheres e de $3,7 \%$ para $21,7 \%$ nos homens (BATISTA MSA, et al., 2017).

No âmbito da política pública brasileira de promoção da saúde da população escolar, destaca-se o PSE lançado pelo Ministério da Saúde e Educação em 2007, que visa promover a formação de alunos da rede no âmbito da atenção básica, através de escolas e médicos Ações integradas e claras entre as equipes da rede pública de ensino e da saúde. O PSE desempenha um papel fundamental no fortalecimento de comportamentos que vinculam saúde, educação e outras redes de serviço social para abordar vulnerabilidades (como a obesidade) que podem prejudicar a saúde de crianças e adolescentes em idade escolar (BATISTA MSA, et al., 2017).

O tracoma é considerado a principal causa infecciosa de cegueira evitável no mundo. A bactéria Chlamydia trachomatis é um patógeno e se manifesta como uma recorrência crônica da conjuntiva e da córnea, resultando em alterações na cicatriz que podem levar à formação de entropia (inversão da pálpebra), triquíase (Cílios de cabeça para baixo) e opacidade da córnea, eventualmente levando à cegueira. O tracoma ocorre em muitas regiões do mundo, incluindo a América Latina. Segundo a Organização Mundial da Saúde (OMS), em 2011, cerca de 325 milhões de pessoas em todo o mundo viviam em áreas endêmicas de tracoma e, só na Europa, não há evidências de que essa doença cause cegueira. Á partir do ano de 2012 a escolas brasileiras passaram a testar alunos através do PSE para identificação do tracoma, e posteriormente inicar os tratamentos preconizados pela OMS (SILVA EJ, et al., 2016).

Como integrante da equipe multiprofissional da estratégia saúde da família, o enfermeiro é responsável pelas atividades educativas do PSE. Ele pode trabalhar com a comunidade escolar para desenvolver diferentes sugestões. Ações educativas cuidadosamente planejadas e implementadas para promover a vacinação contra o Papilomavírus Humano (HPV) têm a função de ampliar o conhecimento pessoal, gerar atitudes adequadas e facilitar mudanças de comportamento, podendo ser uma importante ferramenta para 0 
sucesso de campanhas de vacinação nas escolas e na sociedade, a fim de combater vírus e diversos tipos de tumores. A escola é considerada um ambiente fértil para este tipo de atividades educativas, pois é possível convocar diferentes participantes para fortalecer a vacinação e ter a capacidade de estimular as pessoas a mudarem a realidade, gerando benefícios pessoais e coletivos (SILVA PMC, et al., 2018).

A educação sexual configura-se como uma estratégia para prevenir problemas relacionados ao desenvolvimento do comportamento sexual e gravidez indesejada na adolescência. O PSE promove de alguma forma a implementação de um plano de educação preventiva claro no sistema educacional nacional Junto com o sistema único de saúde, unindo questões relacionadas a sexo, saúde sexual e saúde reprodutiva. Além disso, a experiência de gravidez indesejada na adolescência envolve muitas mudanças no que concerne à gestantes e seu ambiente familiar elevam a uma incompatibilidade e tornam a família e os adolescentes menos propensos a reorganizar seus projetos de vida, às vezes envolvendo interrupções dos estude e desistência do trabalho (SPANIOL C, et al., 2019).

\section{Possíveis novas políticas públicas através do PSE}

As políticas públicas existem para solucionar problemas sociais formados pela ausência de sensibilidade e do Estado, que causam chagas no seio da sociedade e desencadeiam inúmeros outros problemas. Existem políticas públicas atuantes em inúmeros campos, como os programas assistenciais do Governo Federal, por exemplo, agindo no combate à pobreza e a desigualdade social, que são problemas citados na Constituição Federal de 1988 e que devem ser preconizados pelos órgãos públicos. Ações públicas na área da educação e saúde acontecem também por determinação constitucional, já que ambas são direitos fundamentais e devem ser fornecidas pelo Estado (DALFIOR ET, et al., 2015).

A Carta Magna de 1988 aponta que:

"Art. 196. A saúde é direito de todos e dever do Estado, garantido mediante políticas sociais e econômicas que visem à redução do risco de doença e de outros agravos e ao acesso universal e igualitário às ações e serviços para sua promoção, proteção e recuperação.

Art. 197. São de relevância pública as ações e serviços de saúde, cabendo ao Poder Público dispor, nos termos da lei, sobre sua regulamentação, fiscalização e controle, devendo sua execução ser feita diretamente ou através de terceiros e, também, por pessoa física ou jurídica de direito privado.

Art. 205. A educação, direito de todos e dever do Estado e da família, será promovida e incentivada com a colaboração da sociedade, visando ao pleno desenvolvimento da pessoa, seu preparo para o exercício da cidadania e sua qualificação para o trabalho" (BRASIL, 1988).

Assim como todas as áreas do serviço público brasileiro, os programas de saúde apontam problemas e deficiências. Algumas pela má gestão e mau desenvolvimento de ações, outros pela ausência de investimento e sensibilidade para iniciar projetos que atendam essas áreas. O PSE atua em inúmeros campos buscando promover saúde nas fases iniciais de vida dos brasileiros, através de uma união da Atenção Básica com a escola. Muitas questões são trabalhadas através do programa que existe desde 2007 lançado pelo Ministério da Saúde, entretanto algumas necessidades ainda não passam pela chancela do programa. Pode-se apontar como exemplo programas voltados à saúde mental do aluno, que apesar de ser amparado por legislações, não recebem a devida atenção (CID MFB, et al., 2019).

Inúmeras questões referentes a inclusão perpassam pela tutela do Estado e acabam na invisibilidade por influência de outras bandeiras. Dentro do ambiente escolar, espaços são muitas vezes inacessíveis para pessoas com algum tipo de deficiência, e por muito tempo foi preferível isolar essas pessoas ao invés de incluí-las no mesmo ambiente dos alunos que não são portadores de deficiências com alto grau de debilidade. Promover inclusão é uma forma de garantir saúde mental e construir uma escola mais ampla as necessidades sociais, tornando-a um espaço democratizado, para todos, com alta intensidade de preparo para as futuras gerações e capaz de resolver problemas sociais, que de certa forma se tornaram gravíssimos pelo aumento da sensibilidade social para com essas bandeiras (BRIANT MEP e OLIVER FC, 2012). 
O PSE não somente atua em questões ligadas à saúde do aluno, mas também na saúde do professor. É muito mais fácil encontrar na literatura esses apontamentos e as políticas em atividade que cobrem ações de saúde discente, mas enquanto isso a saúde do docente não é tão trabalhada e precisa de maior atenção com políticas públicas específicas. Muitas doenças podem ser desenvolvidas no professor pela alta margem de estresse e desgaste no exercício da profissão. Dentre elas, a principal é a depressão, que é causada pelo desestímulo e pela falta do aparato necessário em sala de aula. Doenças como Lesão por Esforço Repetitivo (LER), lesões nas cordas vocais e até consequências pelos descuidos com a própria saúde, ocasionado pela exaustiva jornada de trabalho, se tornam necessários objetivos dos programas de saúde escolar (PENTEADO RZ e NETO SS, 2019).

\section{CONSIDERAÇÕES FINAIS}

Considera-se, portanto, ao concluir esta pesquisa, que o PSE atua em diversas áreas com base na junção de dois direitos básicos, a saúde e a educação. Os objetivos são nobres e elevam o poder de combate a doenças precoces, evidenciando o quão importante é desenvolver práticas saudáveis e cuidados em tenra idade, objetivando uma geração futura com maior qualidade de vida e disposta aos novos cuidados. Entretanto, é importante ressaltar de igual maneira que o programa carece de melhor desenvolvimento e pode atuar em áreas diferentes à fim de aumentar sua inserção na sociedade e promover cada vez mais saúde nas escolas, atuando como programa de saúde à longo prazo, reduzindo a intensidade de doenças e cobrindo lacunas em campos que ainda não são objetos de políticas pelo Estado.

\section{REFERÊNCIAS}

1. ATALIBA P, MOURÃO L. Avaliação de impacto do Programa Saúde nas Escolas. Psicologia Escolar e Educacional, 2018;

2. BATISTA MSA, et al. Ações do Programa Saúde na Escola e da alimentação escolar na prevenção do excesso de peso infantil: experiência no município de Itapevi, São Paulo, Brasil, 2014. Epidemiologia e Serviços de Saúde, 2017; 26(3): 569-578.

3. BRASIL, 1988. Constituição da República Federativa do Brasil: promulgada em 5 de outubro de 1988. 4. ed. São Paulo: Saraiva, 1990.

4. BRASIL. Cadernos de Atenção Básica: Saúde na Escola. 1 nd ed. Brasília: Ministério da Saúde, 2009; 100p.

5. BRASIL. In: PROGRAMA Saúde nas Escolas: Ministério da Educação. Disponível em: http://portal.mec.gov.br/expansao-da-rede-federal/194-secretarias-112877938/secad-educacao-continuada223369541/14578-programa-saude-nas-escolas. Acesso em: de jul. 2020.

6. BRASIL EGM, et al. Promoção da saúde de adolescentes e Programa Saúde na Escola: complexidade na articulação saúde e educação. Rev Esc Enferm USP, 2017; 51(1): 1-9.

7. BRIANT MEP, OLIVER FC. Inclusão de crianças com deficiência na escola regular numa região do município de São Paulo: conhecendo estratégias e ações. Revista Brasileira de Educação Especial, 2012; 18(1): 141-154.

8. CARVALHO FFB. A saúde vai à escola: a promoção da saúde em práticas pedagógicas. Physis, Revista de Saúde Coletiva, 2015; 25(4): 1207-1227.

9. CASEMIROJP, et al. Promover saúde na escola: reflexões a partir de uma revisão sobre saúde escolar na América Latina. Ciência \& Saúde Coletiva, 2014; 19(3): 829-840.

10. CAVALCANTI PB, et al. Programa Saúde na Escola: interpelações sobre ações de educação e saúde no Brasil. Textos \& Contextos (Porto Alegre), 2015; 14(2): 387-402.

11. CERVATO-MANCUSO AM, et al. O papel da alimentação escolar na formação dos hábitos alimentares. Revista Paulista de Pediatria, 2013; 31(3): 324-330.

12. CID MFB, et al. Saúde mental infantil e contexto escolar: as percepções dos educadores. Pro-Posições, 2019; 30(1): $1-8$.

13. DALFIOR ET, et al. Implementação de políticas públicas: metodologia de análise sob o enfoque da política institucional. Saúde Debate, 2015; 39(1): 283-297.

14. FIGUEIREDO TAM, et al. A saúde na escola: um breve resgate histórico. Ciência \& Saúde Coletiva, 2010; 15(2): 397402.

15. GARCIA JM. Saúde Mental na Escola: O que os Educadores Devem Saber. Psico-USF, 2016; 21 (2): 423-425.

16. GUETERRES ECR, et al. Educação em saúde no contexto escolar: estudo de revisão integrativa. Revista Eletrônica Trimestral de Enfermaria, 2017; 46(1): 477-488.

17. LOPES IE, et al. Eixos de ação do Programa Saúde na Escola e Promoção da Saúde: revisão integrativa. Saúde Debate, 2018; 42(118): 773-789.

18. PENTEADO RZ, NETO SS. Mal-estar, sofrimento e adoecimento do professor: de narrativas do trabalho e da cultura docente à docência como profissão. Saúde e Sociedade, 2019; 28(1): 135-153.

19. SILVA EJ, et al. Prevalência de Tracoma em crianças em idade escolar no município de Turmalina, MG. Revista Brasileira de Oftalmologia, 2016; 75(3): 181-184.

20. SILVA PMC, et al. Conhecimento e atitudes sobre o Papilomavírus humano e a vacinação. Escola Anna Nery, 2018; 22(2): 1-7.

21. SPANIOL C, et al. Gravidez na adolescência e educação sexual: percepções de alunas do ensino médio de um município da Serra Catarinense. Cadernos de Pós-Graduação em Distúrbios do Desenvolvimento, 2019; 19 (2): 61-83. 